\title{
Infecções sexualmente transmissíveis na população carcerária de Alfenas-MG: estudo de prevalência e intervenção educacional
}

\author{
Sexually transmitted infections in the prison \\ population of Alfenas-MG: prevalence study and \\ educational intervention
}

\section{Victor Hugo Tomazela' ${ }^{1}$ (1) Rafael Clemente ${ }^{2}$ (1) Sara Reis de Paula 3 (1) Luisa Patrícia Fogarolli de Carvalho ${ }^{4}$ (1)} 'Autor para correspondência. Universidade Federal de Alfenas (Alfenas). Minas Gerais, Brasil. victor.h.tomazela@gmail.com
${ }^{2-4}$ Universidade Federal de Alfenas (Alfenas). Minas Gerais, Brasil. clemente.rafael@gmail.com, sarareisdepaula@hotmail.com, luisa.fogarolli@gmail.com

RESUMO | INTRODUÇÃo: As Infecções Sexualmente Transmissíveis (IST's) são transmitidas principalmente pelo sexo sem proteção e pelo compartilhamento de perfurocortantes. É tema delicado quando tratado no contexto dos presídios e das Associações de Proteção e Assistência ao Condenado (APAC'S). OBJETIVOS: O objetivo geral foi analisar as relações entre: o encarcerado, seu conhecimento acerca das IST's e sua condição de vulnerabilidade. METODOLOGIA: Trata-se de um estudo transversal e de intervenção educacional. Na análise dos prontuários foram pesquisadas as sorologias (FTAabs IgG, HbsAg, Anti-HCV e Anti-HIV). O estudo de intervenção foi realizado em 3 etapas: $1^{\mathrm{a}}$ - questionário pré-teste; $2^{\mathrm{a}}$ - aula expositiva; e $3^{a}$ - questionário pós-teste. RESULTADOS: Dos 151 prontuários, identificaram-se 3 pessoas HIV positivo, $4 \mathrm{HCV}$ positivo e 8 FTAabs IgG positivo. Na APAC, foi identificado 1 FTAabs IgG positivo. Dos 58 recuperandos da APAC, 34,5\% não acessaram o ensino médio e $37,5 \%$ utilizam preservativos sempre. No pré-teste, a maioria assinalou que a transmissão das IST's se dava pelo compartilhamento de perfurocortantes e pelo sexo desprotegido. No pós-teste notamos ganho de conhecimento na maioria das questões. CONCLUSÃO: Concluindo, a literatura e nossos resultados mostraram maior prevalência de IST's nos presídios. Ademais, nota-se que a baixa escolaridade está relacionada às práticas de risco. Além disso, o compartilhamento de apetrechos para o uso de drogas, realização de tatuagens e o sexo sem condom é considerável. Também foram observadas outros contratempos contribuintes para a alta prevalência de IST's. Por fim, é necessário aumentar os investimentos em politicas públicas voltadas à saúde da população carcerária.

PALAVRAS-CHAVE: Infecções Sexualmente Transmissíveis. Populações vulneráveis. Prisioneiros. Prisões.
ABSTRACT | INTRODUCTION: Sexually Transmitted Infections (STIS) are transmitted primarily through unprotected sex and by sharing of piercings. It is a delicate subject when treated in the context of prisons and the Associations of Protection and Assistance to the Convicted (APACs). OBJECTIVES: The overall objective was to analyze the relationships between: the prisoner, his knowledge about STIs and his condition of vulnerability. METHODOLOGY: This is a cross-sectional study and educational intervention. In the analysis of the promptuaries, serologies (FTAabs-IgG, HbsAg, Anti-HCV and Anti-HIV) were researched. The intervention study was carried out in 3 stages: 1 st pre-test questionnaire; 2 nd lecture; and 3rd post-test questionnaire. RESULTS: From 151 promptuaries, 3 HIV positive, 4 HCV positive, and 8 FTAabs-IgG positive were identified. In APAC, 1 FTAabs-IgG positive was identified. Of the 58 APAC students, $34.5 \%$ did not access high school and $37.5 \%$ always use condoms. In the pre-test, the majority signalled that the STIs transmission was due to the sharing of punctures and unprotected sex. In the post-test we noted a gain of knowledge in most of the questions. CONCLUSION: The literature and our results showed a higher prevalence of STIs in prisons. It is noted that low schooling is related to risk practices. In addition, the sharing of tools for the drug consumption, the realization of tattoos and the unprotected sex is remarkable. Other setbacks contributing to the high prevalence of STIs have also been observed. Finally, it is necessary to increase the investments in policies directed to the prison population health.

KEYWORDS: Sexually Transmitted Infections. Vulnerable populations. Prisoners. Prisons. 


\section{Introdução}

Na literatura encontram-se poucos estudos acerca da possibilidade de capacitação dos indivíduos privados de liberdade através da educação em saúde, como forma de reduzir a prevalência de IST's nas instituições e aprimorar o meio social. Um dos porquês é a ideologia da "periculosidade" que cerceia essa população, traduzindo em pouca disposição de esforços para que o preso tenha acesso à saúde, educação, já que não seria "merecedor"24. Apesar das limitações, acreditamos que a intervenção educacional é a chave do processo de transformação. Portanto, mostramos aqui o impacto das ações educativas em uma população específica da APAC de Alfenas-MG, ressaltando a magnitude do problema na atualidade.

As Infecções Sexualmente Transmissíveis (IST's) são transmitidas principalmente por meio do contato sexual (oral, vaginal, anal) sem o uso de condom. Podem também ser transmitidas via parenteral (compartiIhamento de perfurocortantes) e verticalmente, por gestantes infectadas ${ }^{23}$.

As Associações de Proteção e Assistência ao Condenado (APACs) são instituições as quais visam a inserção do detento na sociedade. Com suas particularidades, visam promover a educação, o trabalho e a convivência sem a exaltação do crime. O detento do Presídio que apresenta boa conduta, por um tempo mínimo, é convidado a ser transferido para a APAC, onde é chamado de "recuperando" e deve respeitar as regras da instituição, caso o contrário, retorna ao Presídio.

Tanto os presidiários como os recuperandos fazem parte de uma população vulnerável ao contágio pelas IST's devido aos comportamentos de alto risco, como o compartilhamento de perfurocortantes e as relações sexuais sem condom. Essas práticas são prevalentes, visto o condicionamento em lugar fechado e insalubre, como também o baixo nível socioeconômico e educacional dos detentos. Soma-se a isso o fato de muitos indivíduos admitidos nessas instituições já trazerem alguma IST adquirida previamente, dessa forma, pode-se esperar que os números referentes à prevalência de IST's sejam similares ou maiores do que os números presentes na sociedade em geral ${ }^{1}$.
A educação na saúde coletiva possibilita, além do acesso e a disseminação da informação, o exercício da cidadania e a responsabilidade social das instituições 25 . Seu impacto na transformação do meio social alvo quando aplicadas por equipes multiprofissionais e interdisciplinares é indubitável, como visto no decorrer do Programa "Viva Mais" em Uberlândia/MG e do projeto "Boas Mãos" em Campinas/SP25. Dessa forma, intervir educacionalmente no ambiente prisional seria extremamente benéfico.

\section{Revisão da Literatura}

A literatura internacional nos evidencia as correlações entre: baixo nível socioeconômico, ambiente prisional e práticas de risco. Um estudo realizado em um presídio feminino nas Filipinas demonstrou que das 100 mulheres selecionadas, $25 \%$ já estavam infectadas por alguma IST antes de serem admitidas no presídio, 54\% não acreditavam que o uso do preservativo realmente protegia, $60 \%$ desconhecia o fato de não haver cura para o HIV e $69 \%$ não sabia que o uso de preservativos durante o sexo reduz as chances de adquirir a doença ${ }^{2}$. Um estudo feito em Taiwan com 910 detentos usuários de drogas injetáveis identificou que 82,1\% dos usuários portadores do HIV e 70,5\% dos usuários não portadores do HIV entendiam que o preservativo não era necessário para prevenção durante o sexo oral, além disso $82,1 \%$ dos portadores de HIV e $83,4 \%$ dos não portadores acreditavam que mosquitos eram capazes de transmitir o HIV. Dos usuários de drogas injetáveis com HIV, $26 \%$ relataram nunca fazer uso de preservativos e $78 \%$ deles afirmaram compartilhar agulhas "as vezes" . No Quirguistão, um estudo com 368 pessoas mostrou que, dos detentos portadores do HIV $(10,3 \%)$, mais da metade $(53,5 \%)$ não tinham conhecimento do diagnóstico até então. Também foi relatado que quase um terço havia iniciado lá o uso de drogas injetáveis, sendo que desses, $86,2 \%$ já haviam injetado drogas dentro da prisão e que $64,2 \%$ compartilhavam seringas com os demais. Além da alta prevalência de HIV, a prevalência de HCV foi de $49,7 \%$, sífilis $19,2 \%$ e HBV $6,2 \%$. 
Os números referentes à prevalência das IST's e às práticas de risco abordadas nos presídios brasileiros mostram-nos a precária situação penitenciária nacional. O Brasil encarcera mais pessoas do que qualquer outro país na América Latina ${ }^{5}$ e conta com a quarta maior população penitenciária do mundo, perdendo apenas para os Estados Unidos, China e Rússia ${ }^{6}$. A população carcerária brasileira é composta, em sua maioria, por adultos jovens. Grande parte desses são homens, pobres, condenados pelos crimes de furto e roubo, pouco alfabetizados e sem profissão definida anteriormente ao encarceramento ${ }^{7}$.

Em um estudo realizado em uma prisão feminina de São Paulo - SP, entre os meses de agosto e outubro de 2000, foi realizado sorologia anti-HIV em 267 detentas e, entre estas mulheres, $37(13,9 \%)$ eram soropositivas $^{8}$. Em outro estudo, realizado em novembro de 2013 na penitenciária feminina de Teresina com 131 mulheres, a prevalência de positividade do marcador sorológico para sífilis foi observada em 33 muIheres, $25,2 \%$ da amostra ${ }^{9}$. Entre a população masculina da Penitenciária de Ribeirão Preto, um estudo realizado no período de maio a agosto de $2003 \mathrm{com}$ 333 presidiários, relatou a prevalência total de infecção pelo HBV de $19,5 \%$, sendo identificado como fatores de risco: tatuagem 36\%, antecedente de IST's 34\% e relação sexual com usuário de droga ilícita $45,7 \%^{10}$. No Centro de Ressocialização Feminino em Rio Claro, foram analisados os resultados laboratoriais de 225 amostras de soro das reeducandas entre março de 2003 e junho de 2004. A prevalência encontrada para HIV foi de 3,6 \%, de Sífilis 3,0\%, de anti-HCV 7,6 $\%$ e dos marcadores HBsAg, anti-HBsAg e anti-HBc, isoladamente, de $0,9 \%, 13,8 \%$ e $2,2 \%$, respectivamente ${ }^{19}$. Em um trabalho brasileiro com 58 mulheres encarceradas, os dados mostraram que $44,8 \%$ delas acreditam que as IST's surgiram como resultado do comportamento homossexual; $72,4 \%$ que os insetos hematófagos podem transmitir IST's; $36,2 \%$ que o sexo anal apresenta um menor risco de transmissão; e $37,9 \%$ entendem que tomar a pílula anticoncepcional conforme orientação médica serve como principal medida de proteção contra IST's $\mathrm{s}^{11}$. Estudo realizado em Teresina com 131 detentas identificou como comportamento de alto risco: o consumo de álcool $(44,3 \%)$ e drogas $(40,5 \%)$ antes das relações sexuais; o sexo sem proteção $(37,4 \%)$; e o baixo conhecimento sobre a transmissão de doenças $(60,3 \%)^{9}$. Do estudo realizado em 12 unidades penais no Piauí, com 2131 participantes destacou-se que, dos casos reagentes para hepatite $B, 54,5 \%$ afirmaram compartilhar materiais perfurocortantes na prisão; $63,3 \%$ utilizavam bebida alcoólica e outras drogas antes das relações sexuais; e apenas $18,1 \%$ deles sabiam algo sobre as formas de infecção ${ }^{12}$.

Diante disso, fica evidente que a prevalência das IST's é maior em situações de privação de liberdade, uma vez que na população geral a prevalência de HIV é de $0,4 \% \frac{13}{13}$, de hepatite B $0,37 \%^{14}$ e de hepatite $\mathrm{C} 0,7 \% \%^{15}$.

Estudos afirmam que intervenções para prevenir, identificar e tratar essas doenças na prisão são pobremente implementadas, principalmente em países de baixa e média renda ${ }^{16}$. É sabido que existem metas e planos governamentais de atuação direta sobre a prevenção e tratamento das IST's no sistema prisional, como o Plano Nacional de Saúde no Sistema Penitenciário que, dentre outras, possui a responsabilidade de auxiliar no diagnóstico, no aconselhamento e no tratamento de IST/HIV/AIDS ${ }^{-}$. Contudo, os altos índices de IST's em presídios brasileiros ainda chamam atenção.

\section{Materiais e Métodos}

Trata-se de um estudo misto: estudo epidemiológico transversal (análise de prontuários) e ensaio de intervenção (educação em saúde). Objetivo geral: analisar as relações entre: o encarcerado, seu conhecimento acerca das IST's e sua condição de vulnerabilidade. Visando isso, têm-se como objetivos específicos: quantificar a prevalência de Hepatite B e C, HIV e Sífilis entre os reclusos; questioná-los sobre as práticas de risco e suas condições socioeconômicas, promover educação em saúde; testar seus conhecimentos prévios; e avaliar o ganho de conhecimento acerca das IST's.

\section{Estudo epidemiológico transversal}

A análise dos prontuários na APAC $(\mathrm{N}=61)$ e no Presídio $(N=520)$ ocorreu no período de julho/2017 a dezembro/2017, onde foram analisadas as sorologias (FTAabs IgG, HbsAg, Anti-HCV e Anti-HIV) 


\section{Ensaio de intervenção}

O ensaio de intervenção foi realizado em 3 etapas: $1 .^{a}$ - questionário pré-teste; $2 .^{a}$ - aula expositiva; e $3 .^{a}$ - questionário pós-teste. O questionário pré-teste foi aplicado no período de dezembro/2017 a março/2018 com 61 recuperandos da APAC. Continha questões de múltipla escolha, referentes às características sociodemográficas, ao uso de drogas, à exposição parenteral, às práticas sexuais e às informações sobre HIV/AIDS, Hepatite B, Hepatite C e Sífilis. As aulas expositivas foram realizadas no período de abril/2018 a maio/2018 na APAC. Essas tiveram duração de aproximadamente 2 horas, abrangendo didaticamente, com imagens, diagramas e textos, os assuntos: formas de transmissão e prevenção, sintomatologia e evolução, práticas de risco, tratamento e profilaxia sobre Sífilis, HIV/AIDS, Hepatite B e Hepatite C. Após as aulas, foram abertas sessões de perguntas e respostas para o esclarecimento de dúvidas remanescentes. Após dois meses da realização das aulas, no período de julho/2018 a setembro/2018, foi realizada a $3^{a}$ etapa, com aplicação do questionário pósteste entre 58 recuperandos da APAC. O pós-teste era igual ao pré-teste e todos os participantes leram e responderam seus questionários sozinhos, sendo que alguns pediram auxílios pontuais aos pesquisadores para que esclarecessem o significado de algumas palavras. Os dados foram analisados através de estatística descritiva. A construção de tabelas e gráficos foi realizada através do software Excel Office ${ }^{\circledR}$.

A pesquisa encontra-se em consonância com a Resolução 466/2012 do Conselho Nacional de Saúde do Ministério da Saúde e foi aprovado pelo Comitê de Ética em Pesquisa da Universidade Federal de Alfenas - parecer de aprovação do Comitê de Ética e Pesquisa no 2.195.931 (CAAE 68613617.8.0000.5142).

Foram excluídos do estudo os que não participaram de todas as atividades propostas e os que se recusaram a assinar o Termo de Consentimento Livre e Esclarecido (TCLE).

\section{Resultados e discussão}

\section{Prevalência de IST's na APAC e no Presídio}

Foram analisados 61 prontuários da APAC, encontrando a prevalência de Sífilis 1,64\% IC95\% (0,29\% - 8,72\%). Também foram analisados 520 prontuários do Presídio. Desses somente 151 continham exames sorológicos, sendo a prevalência de HIV 3,31\%, IC95\% (1,42\% - 7,52\%) de HCV 2,65\% IC95\% (1,03\% - 6,61\%) e de Sífilis 5,96\% IC95\% (3,17\% - 10,94\%).

Ao avaliar, na literatura nacional e internacional, a prevalência de IST's no sistema carcerário, encontramos os seguintes intervalos de prevalências:

Tabela 1. Comparação entre as prevalências do Presídio de Alfenas, APAC de Alfenas e na literatura mundial

\begin{tabular}{llll}
\hline & HIV & Hepatite C & Sifilis \\
\hline Presídio de Alfenas & $3,31 \%$ & $2,64 \%$ & $5,96 \%$ \\
APAC de Alfenas & - & - & $1,62 \%$ \\
Literatura mundial & $0,04 \%^{17}-13,6 \%^{3}$ & $4,5 \%{ }^{18}-18,7 \%^{9}$ & $3 \%{ }^{19}-25,2 \%^{17}$ \\
\hline
\end{tabular}

Fonte: Os autores (2018). 
Como observado na Tabela 1, os dados obtidos são comparáveis percentualmente às prevalências encontradas na literatura. Além disso, as prevalências encontradas no sistema prisional de Alfenas também são maiores que as da população em geral. Seguem exemplos de prevalências de IST's na população comum: HIV 0,4\%13; Hepatite

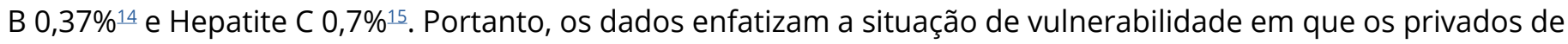
liberdade se encontram.

A Tabela 2 nos mostra a comparação entre a prevalência de Sífilis na APAC e no Presídio, colocando esse último como fator de risco para o contágio com a IST:

Tabela 2. Comparação entre a prevalência de Sífilis entre a APAC (Grupo controle) e o Presídio (Grupo Expostos)

\begin{tabular}{llll}
\hline & & Portador de Sífilis & Total \\
Presídio & Sim & Não & 151 \\
APAC & 9 & 142 & 61 \\
Total & 1 & 60 & 189 \\
\hline
\end{tabular}

Fonte: Os autores (2018).

Analisando a Tabela 2, a APAC apresenta notavelmente uma menor prevalência de Sífilis ao compararmos com o Presídio. No entanto, não se obteve significância na associação estatística. (Razão de Prevalência: 3,80. IC95\% $(0,47-30,68))$.

\section{Nível educacional na APAC}

Gráfico 1. Nível educacional dos recuperandos da APAC de Alfenas-MG

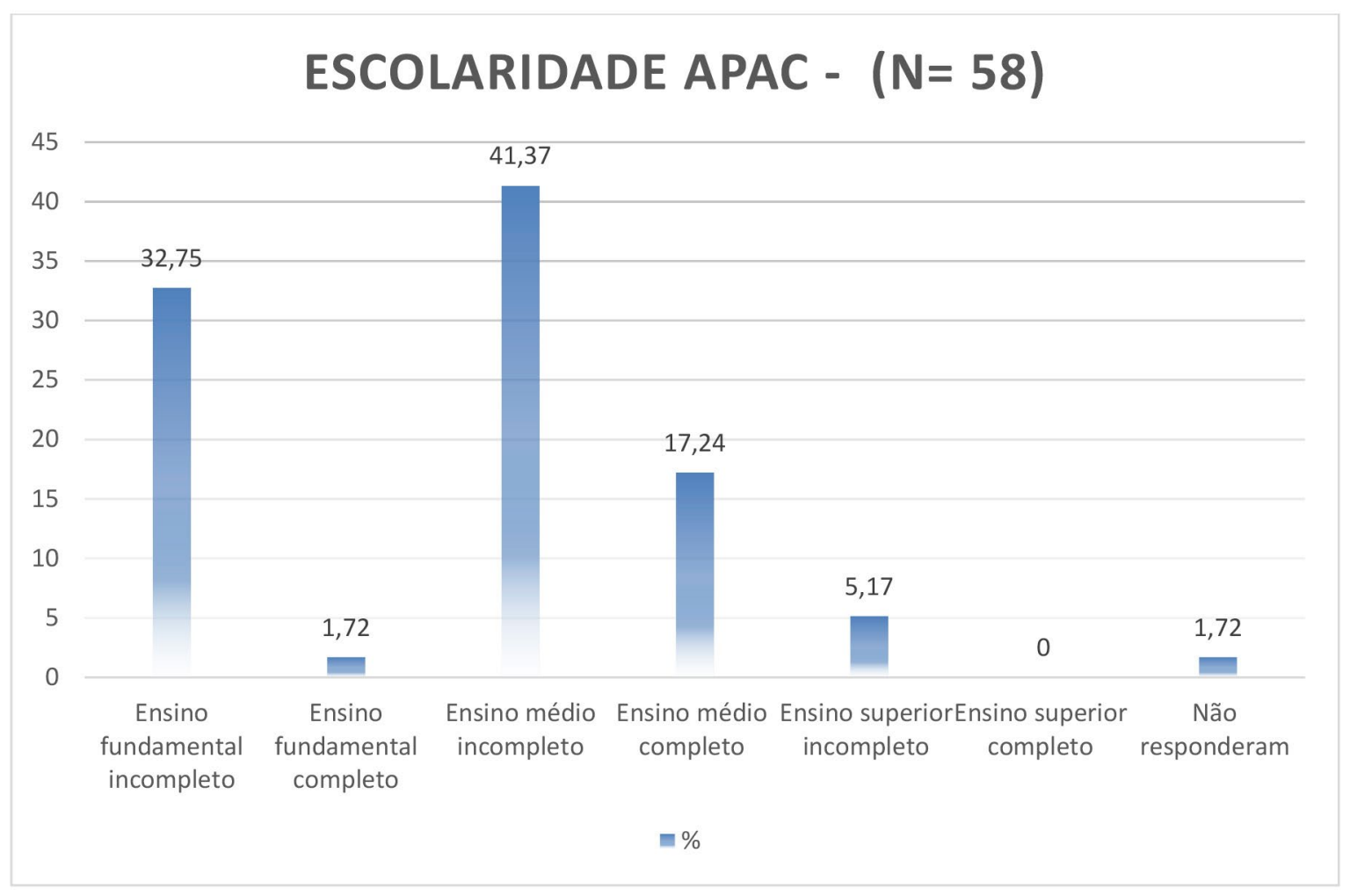

Fonte: Os autores (2018). 
Em nossa amostra de 58 recuperandos da APAC masculina, 34,47\% IC95\% (23,5\% - 47,3\%) ainda não acessaram o ensino médio, sendo que desses, somente $1,72 \%$ IC95\% (0,3\% - 9,1\%) concluiu o ensino fundamental. Dos que se encontram no ensino médio, tendo ou não concluído, temos 58,61\% IC95\% (45,8\% - 70,37\%) da população entrevistada. Nenhum dos entrevistados possui ensino superior completo.

O baixo grau de escolaridade está intimamente relacionado com as práticas de risco para infecção por IST's, bem como com o grau de conhecimento apresentado sobre as IST's abordadas nesse estudo. De acordo com o Levantamento Nacional de Informações Penitenciárias de 2016, da amostra de 482.645 pessoas privadas de liberdade, $75 \%$ ainda não acessou o ensino médio, tendo concluído, no máximo, o ensino fundamental. Entre a população que se encontra no ensino médio, tendo concluído ou não esta etapa da educação formal, temos apenas $24 \% 20$.

\section{Ganho de conhecimento (APAC)}

O chamado "ganho de conhecimento" [(C-1) no Quadro 1] é uma comparação em porcentagem entre as taxas das respostas dos questionários pré-teste e pós-teste, realizados com a população masculina da APAC, sendo que o pré-teste contou com a participação de 61 recuperandos e o pós-teste 58. Todos os participantes participaram após a realização do préteste da aula expositiva teórica. Foram excluídos 3 participantes, pois não frequentaram a aula expositiva. No Quadro 1, apresentamos algumas das questões presentes nos questionários pré e pós teste e qual a porcentagem dos recuperandos que assinalaramnas como verdadeiras, tanto antes da aula expositiva como depois.

Quadro 1. Comparação das questões entre o pré e o pós-teste. "(C-1) = Ganho de conhecimento"

\begin{tabular}{|c|c|c|c|c|c|c|}
\hline Questões respondidas como verdadeiras. & $\begin{array}{l}\text { Números a } \\
\text { resp }\end{array}$ & $\begin{array}{l}\text { solutos de } \\
\text { stas }\end{array}$ & & & & \\
\hline Questão & $\begin{array}{l}\text { Pré Teste } \\
(\mathrm{N}=61)\end{array}$ & $\begin{array}{l}\text { Pós Teste } \\
(\mathrm{N}=58)\end{array}$ & $\begin{array}{l}\% \text { Pré Teste } \\
\text { (A) }\end{array}$ & $\begin{array}{l}\% \text { Pós } \\
\text { Teste (B) }\end{array}$ & $C=A-B$ & $\mathrm{C}-1$ \\
\hline “Hepatite B é transmitida pelo ar?” & 12 & 5 & $19,7 \%$ & $8,7 \%$ & 0,44 & $-56 \%$ \\
\hline “Hepatite B é transmitida pelo sangue?” & 32 & 41 & $52,4 \%$ & $70,7 \%$ & 1,35 & $35 \%$ \\
\hline $\begin{array}{l}\text { "Sexo anal sem preservativos pode pegar } \\
\text { Hepatite C?" }\end{array}$ & 37 & 45 & $60,6 \%$ & $77,6 \%$ & 1,28 & $28 \%$ \\
\hline $\begin{array}{l}\text { "Ferida genital indolor pode ser sinal de } \\
\text { sifilis?" }\end{array}$ & 15 & 29 & $24,6 \%$ & $50,0 \%$ & 2,03 & $103 \%$ \\
\hline "A sífilis é transmitida pelo sexo oral?" & 29 & 38 & $47,5 \%$ & $65,5 \%$ & 1,38 & $38 \%$ \\
\hline $\begin{array}{l}\text { "Ao engolir sêmen pode ser contaminado } \\
\text { pelo HIV?" }\end{array}$ & 10 & 25 & $16,4 \%$ & $43,1 \%$ & 2,63 & $163 \%$ \\
\hline $\begin{array}{l}\text { “O uso de camisinha impede a } \\
\text { transmissão da doença durante o sexo?" }\end{array}$ & 53 & 51 & $87,0 \%$ & $88,0 \%$ & 1,01 & $1 \%$ \\
\hline $\begin{array}{l}\text { "Compartilhar alicates e cortadores de } \\
\text { unha e seringas oferecem o risco de } \\
\text { contaminação pelo HIV?" }\end{array}$ & 47 & 48 & $77,0 \%$ & $83,0 \%$ & 1,08 & $8 \%$ \\
\hline $\begin{array}{l}\text { “Não é possível "pegar" Hepatite C apenas } \\
\text { conversando com alguém contaminado" }\end{array}$ & 54 & 52 & $88,5 \%$ & $89,6 \%$ & 1,01 & $1 \%$ \\
\hline
\end{tabular}


Ficou nítido que o entendimento dos conteúdos ministrados em aulas foi satisfatório pelos recuperandos, como se pode observar, por exemplo, na questão: "Ao engolir sêmen pode ser contaminado pelo HIV?", onde obtivemos uma porcentagem de acertos de $163 \%$ a mais no pós-teste comparando com o pré-teste; como também na questão "Ferida genital indolor pode ser sinal de sífilis?", mostrandonos $103 \%$ a mais. No entanto, houve questões que não apresentaram uma variação expressiva, como, por exemplo, na questão: "O uso de camisinha impede a transmissão da doença durante o sexo?", mostrando-nos um conhecimento prévio relevante. Essas informações, em conjunto, nos revelam que as atividades educacionais devem ser cada vez mais incentivadas e realizadas, a fim de combater a desinformação e melhorar a prevenção destas populações contra as IST's.

\section{Análise dos comportamentos de risco}

\section{Uso de drogas}

Na APAC, dos 61 recuperandos, 67,2\% IC95\% (54,7\% $77,6 \%)$ fazem uso de drogas, sendo que $85,4 \%$ IC95\% $(71,5 \%$ - 93,12\%) fazem uso de drogas inalatórias e $14,6 \%$ IC95\% (6,9\% - 28,4\%) de drogas injetáveis. É fato que a relação sexual com usuário de drogas aumenta o risco de contrair IST's. Temos dois fatores: o primeiro é o consumo de drogas de qualquer natureza antes das relações sexuais, pois elas estão relacionadas ao não uso de preservativos ${ }^{21}$. Outro estudo no Piauí12 corrobora com esse fato, visto que dos 2131 detentos participantes, 63,3\% afirmaram utilizar bebida alcoólica e outras drogas antes das relações sexuais. O segundo fator é o compartilhamento de materiais para o uso de drogas, sendo os perfurocortantes de maior risco e em segundo lugar o compartilhamento de apetrechos e matérias no uso não parenteral, como canudos para o uso de cocaína, cachimbos etc ${ }^{21}$.

\section{Uso de preservativos}

Um fator preocupante é a baixa aderência ao uso de preservativos nos presídios. Em nosso estudo encontramos que, na APAC masculina, dos 61 indivíduos, $52 \%$ IC95\% (40,1\% - 64,5\%) utilizam preservativos durante a relação sexual, sendo que desses somente $37,5 \%$ IC95\% (22,9\% - 54,7\%) utilizam sempre. Dentre os motivos de não utilizarem o preservativo, respondido por 58 indivíduos o mais relevante foi que "não gostam, pois atrapalha" $36,2 \%$ IC95\% (25\% - 49\%). Um estudo realizado em um presídio feminino nas Filipinas demonstrou que das 100 mulheres, 54\% não acreditavam que o uso do preservativo realmente protegia e $69 \%$ não sabia que o uso de preservativos durante o sexo reduz as chances de adquirir a doença ${ }^{2}$. Em um estudo realizado no Piauí, com 2131 participantes destacou-se que somente $27,2 \%$ dos participantes faziam uso de preservativos durante a relação sexual. Dentre os motivos a variável "Não gosta de usar camisinha nas relações" apresentou grande associação ${ }^{12}$.

\section{Realização de tatuagens}

A realização de tatuagens dentro dos presídios representa um comportamento de risco devido ao compartilhamento de perfurocortantes. Em nosso estudo, $26 \%$ IC95\% (16,3\% - 38,3\%) dos 58 recuperandos, disseram tê-la realizada no presídio ou na APAC. Encontramos na literatura índices de igual preocupação. No estudo feito no presídio masculino em Belize no ano de 2005 com 623 presos, 35\% relataram terem feito alguma tatuagem na prisão ${ }^{22}$. Na Hungria, em estudo semelhante com 1553 indivíduos, evidenciou-se que $14,3 \%$ dos detentos haviam feito a última tatuagem no presídio ${ }^{18}$.

Com base nos dados expostos acima, as populações carcerárias carregam consigo um deficit educacional grande, mesmo antes do encarceramento. Os baixos níveis socioeconômicos e de escolaridade destas populações apresentam-se como fatores de risco para as IST's. Ao desconhecerem, em boa parte, os modos de transmissão, prevenção e tratamento, os recuperandos colocam-se em uma posição extremamente vulnerável, ainda mais quando se considera o ambiente no qual estão inseridos. A própria situação do encarceramento eleva ainda mais os riscos de contaminação, através do compartilhamento de instrumentos, como agulhas, cortadores de unha e materiais para uso de drogas. Soma-se a isso os hábitos sexuais dentro de instituições carcerárias, em especial as baixas taxas de uso de preservativos durante a atividade sexual. 


\section{Conclusão}

Como se pode observar, inúmeros fatores encadeiam-se para acentuar a situação de vulnerabilidade a qual essa população está exposta, contribuindo para uma elevada prevalência das IST's.

Esse trabalho apresenta algumas limitações, como: os presidiários podem optar por não realizar as sorologias, dessa forma só 151 de 520 prontuários continham exames sorológicos para as IST's, subestimando a real prevalência; a falta de estrutura para um ambiente de aprendizagem, pois utilizamos uma cela desativada sem espaço suficiente e com pouca luz para as atividades; as aulas expositivas foram ministradas somente uma vez por grupo, contribuindo provavelmente para uma diminuta retenção de conhecimento, sendo necessário nos próximos estudos aumentar a frequência; alguns detentos não estavam interessados e sentiram-se obrigados pela direção da instituição, portanto responderam de forma muito rápida, o que pode contribuir para erros de aferição; a longa distância entre as instituições e a cidade de Alfenas-MG somada à agenda restrita das instituições para as atividades e a dificuldade de contato com a direção contribuíram de forma negativa, alongando demasiadamente a duração da pesquisa e exigindo gastos financeiros consideráveis; a mudança dos diretores do presídio e da APAC junto com duas greves realizadas pelos detentos também contribuíram negativamente na duração da pesquisa.

Portanto, como visto, existem dificuldades e limitações em instituições prisionais, as quais extrapolam as comuns em pesquisas de campo, dificultando $a$ inserção de equipes de saúde e pesquisadores. Tal fato distancia a Academia do sistema prisional e dificulta a realização de diferentes pesquisas e atividades que poderiam ser extremamente benéficas tanto para os privados de liberdade quanto para as próprias instituições.

Acreditamos que o incremento em políticas públicas voltadas à saúde e à educação do detento meIhoraria a prevenção primária, isto é, mais atividades educacionais, melhorias nas condições do ambiente e acompanhamento da saúde do detento. Com isso poderia haver um aumento no nível educacional e condição de saúde, culminando na diminuição das práticas de alto risco e, consequentemente, da prevalência das IST's. No entanto, a formulação de uma política que aumente o acesso à saúde em um ambiente superlotado e insalubre é um processo laborioso e problemático 24 , exigindo uma equipe multiprofissional e interdisciplinar 24,25 .

\section{Contribuições dos autores}

Tomazela VH, Clemente R, Paula SR participou da concepção, delineamento, busca e análise estatística dos dados da pesquisa, interpretação dos resultados e redação do artigo científico. Carvalho LPF participou da concepção, delineamento, interpretação dos resultados e redação do artigo científico.

\section{Conflitos de interesses}

Nenhum conflito financeiro, legal ou político envolvendo terceiros (governo, empresas e fundações privadas, etc.) foi declarado para nenhum aspecto do trabalho submetido (incluindo, mas não se limitando a subvenções e financiamentos, participação em conselho consultivo, desenho de estudo, preparação de manuscrito, análise estatística, etc.).

\section{Referências}

1. Scott N, McBryde E, Kirwan A, Stoové M. Modelling the Impact of Condom Distribution on the Incidence and Prevalence of Sexually Transmitted Infections in an Adult Male Prison System. PLoS One. 2015;10(12):e0144869. doi: 10.1371/journal. pone.0144869

2. Simbulan NP, Aguilar AS, Flanigan T, Cu-Uvin S. High-risk Behaviors and the Prevalence of Sexually Transmitted Diseases Among Women Prisioners at the Women State Penitentiary in Metro Manila. Social Science \& Medicine. 2001;52(4):599-608. doi: 10.1016/s0277-9536(00)00163-5

3. Feng MC, Feng JY, Chen YH, Chang PY, Lu PL. Prevalence and knowledge of sexual transmitted infections, drug abuse, and AIDS among male inmates in a Taiwan prison. Kaohsiung J Med Sci. 2012;28(12):660-6. doi: 10.1016/j.kjms.2012.04.035

4. Azbel L, Polonsky M, Wegman M, Shumskaya N, Kurmanalieva A, Asanov A et al. Intersecting epidemics of HIV, HCV, and syphilis among soon-to-be released prisoners in Kyrgyzstan: Implications for prevention and treatment. Int J Drug Policy. 2016;37:9-20. doi: 10.1016/j.drugpo.2016.06.007

5. Mariner J, Cavallaro J. O Brasil Atrás das Grades. Nova York: Human Rights Watch; 1998.

6. Silva GS, Souza JVS, Silva KJS. Soroprevalência e fatores de risco para o vírus da imunodeficiência humana (HIV), vírus da hepatite $\mathrm{c}(\mathrm{HCV})$, vírus da hepatite b (HBV) e treponema pallidum em presidiários do município de Caruaru-PE [dissertação]. Caruaru (PE): Centro Universitário Tabosa de Almeida; 2017. 
7. Ministério da Saúde. Plano Nacional de Saúde no Sistema Penitenciário [Internet]. 2004. Disponível em: http://bvsms.saude. gov.br/bvs/publicacoes/cartilha_pnssp.pdf

8. Strazza L, Azevedo RS, Carvalho HB. Prevenção do HIV/Aids em uma Penitenciária-modelo feminina de São Paulo-SP, Brasil. DST-J Bras Doenças Sex Transm. 2006;18(4):235-40.

9. Araújo TME, Araujo Filho ACA, Feitosa KVA. Prevalência de sífilis em mulheres do sistema prisional de uma capital do nordeste brasileiro. Rev Eletr Enf.. 2016;17(4). doi: 10.5216/ree.v17i4.28898

10. Coelho HC, Oliveira SAN, Miguel JC, Oliveira MLA, Figueiredo JFC, Perdoná GC et al. Soroprevalência da infecção pelo vírus da Hepatite B em uma prisão brasileira. Rev Bras Epidemiol. 2009;12(2):124-131. doi: 10.1590/\$1415-790X2009000200003

11. Costa ES, Santos JDM, Rocha MRC, Viana LMM, Oliveira DM, Silva BJC et al. Mulheres Encarceradas: Perfil, Sexualidade e Conhecimento Sobre Infecções Sexualmente Transmissíveis. Rev UNINGÁ. 2017;52(1):23-8.

12. Silva AAS, Araújo TME, Teles SA, Magalhães RLB, Andrade ELR. Prevalência de hepatite $B$ e fatores associados em internos de sistema prisional. Acta Paul. Enferm. 2017;30(1):66-72. doi: 10.1590/1982-0194201700010

13. Ministério da Saúde. Boletim Epidemiológico - AIDS/HIV. 5th ed. Brasília (DF): Departamento de Doenças de Condições Crônicas e Infecções Sexualmente Transmissíveis. [Internet]. 2017. Disponível em: http://www.aids.gov.br/pt-br/pub/2017/ boletim-epidemiologico-hivaids-2017

14. Estudo de prevalência de base populacional das infecções pelos vírus das hepatites A, B e C nas capitais do Brasil. BEPA, Bol Epidemiol Paul. 2007; 4(44): 23-24.

15. Ministério da Saúde. Hepatites Virais 2017. Boletim Epidemiológico [Internet]. 2017;48(24). Disponível em: http://giv. org.br/Hepatites-Virais/boletim_hepatites_virais_2017_ms.pdf

16. Dolan K, Wirtz AL, Moazen B, Ndeffo-Mbah M, Galvani A, Kinner AS et al. Global Burden os HIV, Viral Hepatitis, and Tuberculosis in Prisoners and Detainees. Lancet. 2016;388(10049):1089-1102. doi: 10.1016/S0140-6736(16)30466-4
17. Adjei AA, Armah HB, Gbagbo F, Ampofo WK, Boamah I, Adu-Gyamfi $C$ et al. Correlates of HIV, HBV, HCV and syphilis infections among prison inmates and officers in Ghana: A national multicenter study. BMC Infect Dis. 2008;8:33. doi: 10.1186/14712334-8-33

18. Tresó $B$, Barcsay E, Tarján A, Horváth G, Dencs A, Hettmann A et al. Prevalence and correlates of HCV, HVB, and HIV infection among prison inmates and staff, Hungary. J Urban Health. 2012;89(1):108-16. doi: 10.1007/s11524-011-9626-X

19. Berra JAP, Bacetti LB, Buzo AA. Soroprevalência de HIV, Sífilis, Hepatite B e C em Mulheres do Centro de Ressocialização Feminino, Rio Claro, São Paulo. Rev Inst Adolfo Lutz. 2006; 65(2):133-6.

20. Ministério da Justiça e Segurança Pública. Levantamento Nacional de Informações Penitenciárias [Internet]. Brasília, DF: Departamento Penitenciário Nacional; 2017. Disponível em: https://www.conjur.com.br/dl/infopen-levantamento.pdf

21. Reis NB. Conhecimento Sobre HIV/AIDS Entre Usuários de Drogas. [dissertação]. Rio de Janeiro: Fundação Oswaldo Cruz; 2010.

22. Gough E, Edwards P. HIV seroprevalence and associated risk factors among male inmates at the Belize Central Prison. Rev Panam Salud Publica. 2009;25(4):292-9. doi: 10.1590/s102049892009000400002

23. Ministério da Saúde. Infecções Sexualmente Transmissíveis (IST): o que são, quais são e como prevenir [Internet]. 2018 [acesso em 2018 nov 09]. Disponível em: http://antigo.saude.gov. br/saude-de-a-z/infeccoes-sexualmente-transmissiveis-ist

24. Barsaglini R. Do Plano à Política de saúde no sistema prisional: diferenciais, avanços, limites e desafios. Physis. 2016;26(4):14291439. doi: $10.1590 / \mathrm{s} 0103-73312016000300019$

25. Silva MI, Pelazza BB, Souza JH. Relato de experiências de ações educativas para saúde em comunidades socialmente vulneráveis. Diversa Prática. 2016; 3(1):17-40. doi: 10.14393/DPv3n1-2016-49615 